\title{
External and Intraparticle Diffusion of Coumarin 102 with Surfactant in the ODS-silica Gel/water System by Single Microparticle Injection and Confocal Fluorescence Microspectroscopy
}

\author{
Kiyoharu NaKatani ${ }^{\dagger}$ and Emi Matsuta \\ Division of Chemistry, Faculty of Pure and Applied Sciences, University of Tsukuba, 1-1-1 Tennoudai, \\ Ibaraki 305-8571, Japan
}

\begin{abstract}
The release mechanism of coumarin 102 from a single ODS-silica gel microparticle into the water phase in the presence of Triton X-100 was investigated by confocal fluorescence microspectroscopy combined with the single microparticle injection technique. The release rate significantly depended on the Triton X-100 concentration in the water phase and was not limited by diffusion in the pores of the microparticle. The release rate constant was inversely proportional to the microparticle radius squared, indicating that the rate-determining step is the external diffusion between the microparticle and the water phase.
\end{abstract}

Keywords Porous material, ODS-silica gel, diffusion, confocal fluorescence microspectroscopy, surfactant

(Received December 24, 2014; Accepted March 2, 2015; Published June 10, 2015)

\section{Introduction}

Kinetic analysis of mass transfer of a solute between a porous material and the surrounding solution phase is significant for understanding chromatography, solid phase extraction, drug delivery systems, soil remediation, and so forth. ${ }^{1-4}$ The mass transfer in the porous material/solution system has been often analyzed in terms of diffusion in pores of the material..$^{5-10}$ However, analysis of the mass transfer mechanism is generally difficult. As an example, mass transfer in a porous particle/ solution system consists of several elementary processes such as adsorption/desorption at the pore walls, mass transfer in the surrounding solution (external mass transfer), and diffusion in the pore solution (pore diffusion) and along the pore walls (surface diffusion). The overall mass transfer rate depends on the particle size, the interparticle distance, and so on. Direct measurements of the individual elementary processes are necessary for the analysis of the mass transfer mechanism in the porous material/solution system. ${ }^{5,6,9,11,12}$

Mass transfer of a dye between a porous microparticle and the surrounding solution phase was analyzed by single microparticle injection and absorption microspectroscopy. ${ }^{6,13}$ In the single microparticle/solution system, external mass transfer between the microparticle and the bulk solution is steady-state spherical diffusion so that analysis of the external mass transfer is simplified. Using the single microparticle injection and absorption microspectroscopy, time course of the concentration of a water-soluble dye such as rhodamine $6 \mathrm{G}$ and bromophenol blue in the single microparticle was measured and the mass transfer mechanism of the dye between the microparticle and

$\dagger$ To whom correspondence should be addressed.

E-mail: nakatani@chem.tsukuba.ac.jp the solution could be analyzed from the sorption or release rates. ${ }^{14-16}$ In those reports, as the mass transfer rate in the microparticle/solution system was assumed to be limited by the intraparticle diffusion, the intraparticle diffusion coefficient $\left(D_{\mathrm{p}}\right)$ was estimated. When the supposed $D_{\mathrm{p}}$ value was independent of the microparticle diameter $(d)$, the intraparticle diffusion was analyzed in detail on the basis of the pore and surface diffusion model. ${ }^{14-16}$ For mass transfer of perylene as a water-insoluble organic compound in the presence of Triton X-100 (nonionic surfactant) in an ODS-silica gel/water system, however, the supposed $D_{\mathrm{p}}$ value depended on $d$, indicating that the mass transfer rate cannot be analyzed as the intraparticle diffusion. ${ }^{17}$ From the $d$ dependence of mass transfer rate, we concluded that the rate-determining step was the micelle-mediated solubilization of the dye at the microparticle surface. ${ }^{17}$ Kinetic analysis of complicated mass transfer in a porous microparticle/solution system may be difficult to achieve by single microparticle injection and absorption microspectroscopy alone.

In the present report, we have developed confocal fluorescence microspectroscopy combined with the single microparticle injection technique for analysis of the release of coumarin 102, as a poorly water-soluble organic compound, from an ODSsilica gel microparticle into water in the presence of Triton $\mathrm{X}-100$. Since the confocal fluorescence microspectroscopy possesses three-dimensional spatial resolution, ${ }^{11,18,19}$ different from absorption microspectroscopy, distribution of the fluorescent dye in the microparticle interior was analyzed and the mass transfer behavior of the dye could be directly observed. The kinetic analysis of the mass transfer of the organic compound in the porous microparticle/water system in the presence of the surfactant will be important for understanding elementary processes for soil remediation and micellar liquid chromatography. ${ }^{20-23}$ We show the potential of the single microparticle injection and confocal fluorescence 
microspectroscopy and discuss the release mechanism of coumarin 102 from ODS-silica gel into water in the presence of Triton X-100.

\section{Experimental}

Spherical ODS-silica gel (Wako Pure Chemical Industries, Wakosil40C18: $d=30-50 \mu \mathrm{m}$; surface area, $330 \mathrm{~m}^{2} \mathrm{~g}^{-1}$; pore diameter, $12 \mathrm{~nm}$; pore volume, $\left.1.0 \mathrm{~cm}^{3} \mathrm{~g}^{-1}\right)$, coumarin 102 (Wako Pure Chemical Industries, laser grade), $N, N$ dimethylformamide (Wako Pure Chemical Industries, JIS Special Grade), and Triton X-100 (Sigma-Aldrich, Laboratory Grade, average molar weight: $625 \mathrm{~g} / \mathrm{mol}$ ) were used without further purification. Water was used after deionization and distillation (Yamato Scientific, Auto Still WG203). ODS-silica gel was soaked in a dimethyformamide/water (1:1) solution and stored for over a day.

For release rate measurements by confocal fluorescence microspectroscopy, several ODS-silica gel microparticles in the dimethyformamide/water solution were dispersed into an aqueous solution containing coumarin $102\left(\sim 10^{-6} \mathrm{~mol} \mathrm{dm}^{-3}\right)$ and Triton X-100 $\left(0-2 \mathrm{mmol} \mathrm{dm}^{-3}\right)$ and left for over a week at $25^{\circ} \mathrm{C}$. Using a microcapillary manipulation/injection system (Narishige, MN-151/IM-16), the single microparticle sorbing the coumarin 102 and Triton X-100 was injected into an aqueous solution containing Triton X-100 with the same concentration in the aqueous solution before injection, and then three-dimensional spatially resolved fluorescence measurements of the single microparticle were performed by a confocal fluorescence microscope (Olympus, FV 1000-D) at room temperature $\left(\sim 25^{\circ} \mathrm{C}\right)$. A CW diode laser beam $(405 \mathrm{~nm}, 0.15 \mathrm{~mW})$ led to the microscope was focused on the microparticle through an objective lens (Olympus, UPLSAPO 60xH) and fluorescence of $425-525 \mathrm{~nm}$ was detected. Confocal aperture size was $100 \mu \mathrm{m}$.

For distribution coefficient measurements, several ODS-silica gel microparticles in the dimethyformamide/water solution were dispersed into an aqueous solution containing coumarin 102 $\left(10^{-6}-10^{-5} \mathrm{~mol} \mathrm{dm}^{-3}\right)$ and Triton X-100 $\left(0-2 \mathrm{mmol} \mathrm{dm}^{-3}\right)$ using the microcapillary manipulation/injection system, and left for over a week at $25^{\circ} \mathrm{C}$. The distribution coefficient $\left(K_{\mathrm{e}}\right)$ was determined through the dye concentration measurements in the microparticle and the water phase. Absorption spectra of the dye in the individual microparticles and the water phase were measured by absorption microspectroscopy and conventional absorption spectroscopy, respectively. ${ }^{6}$

\section{Results and Discussion}

Fluorescence intensity profile measurements in a single microparticle

Coumarin 102 has been known as a solvatochromic dye and the fluorescence maximum wavelength $\left(\lambda_{\max }\right)$ of coumarin 102 depends on the microenvironment surrounding the dye. In single ODS-silica gel microparticles, $\lambda_{\max }$ was determined to be $464 \mathrm{~nm}$ in the absence of Triton X-100 and that decreased when increasing the Triton X-100 concentration in the water phase $\left([\text { Triton } \mathrm{X}-100]_{\mathrm{w}}\right)\left(456 \mathrm{~nm}\right.$ at $[\text { Triton } \mathrm{X}-100]_{\mathrm{w}}=0.05$ $\mathrm{mmol} \mathrm{dm}^{-3}$ ). For release rate measurements, coumarin 102, sorbed into an ODS-silica gel microparticle in an aqueous Triton $\mathrm{X}-100$ /coumarin 102 solution, was released into an aqueous Triton X-100 solution of the same [Triton X-100] $]_{\mathrm{w}}$ as that for the sorption without coumarin 102. As $\lambda_{\max }$ was independent of

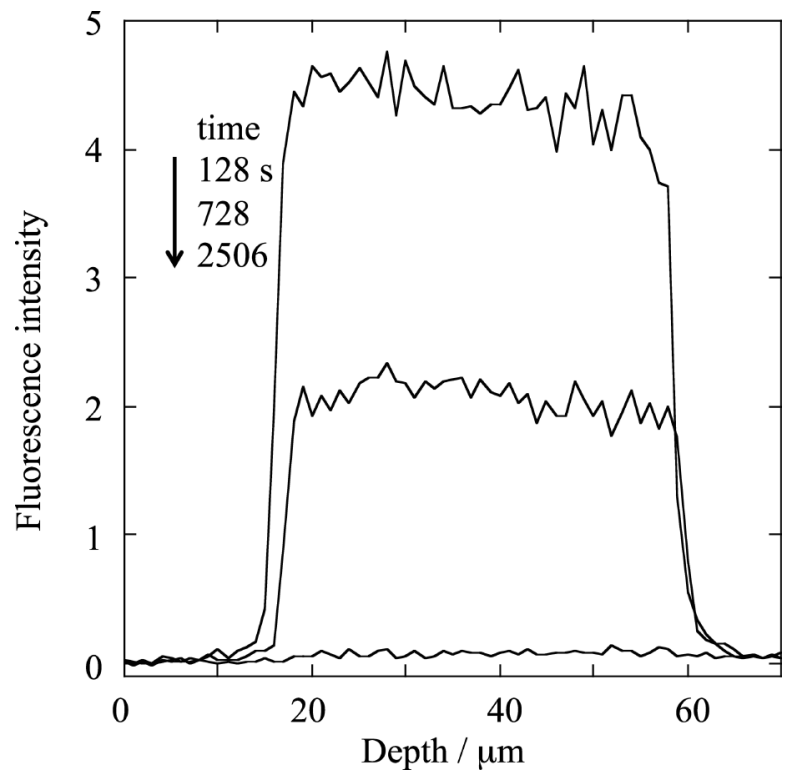

Fig. 1 Time dependence of fluorescence depth profile of coumarin 102 in a single ODS-silica gel microparticle $(d=40 \mu \mathrm{m})$ injected into an aqueous $2 \mathrm{mmol} \mathrm{dm}^{-3}$ Triton X-100 solution.

the release time, the amount of the Triton X-100 sorbed in the ODS-silica gel is expected to be constant during the release rate measurement.

Fluorescence intensity distribution of coumarin 102 in an ODS-silica gel microparticle was measured by the confocal fluorescence microscope. The depth (longitudinal $(Z)$ axis) dependence of the fluorescence intensity of a circle with $4.1 \mu \mathrm{m}$ radius as a lateral ( $X Y$ axes) area was analyzed from lower part to upper part of the spherical microparticle along the central axis. Figure 1 shows the time $(t)$ course of the fluorescence depth profile of the dye in a single microparticle. For the fluorescence intensity profile measurements, the absorbance of the dye in the single microparticle at $405 \mathrm{~nm}$ was less than 0.002 so that the exciting laser light intensity is assumed to be constant, independent of the depth position. The fluorescence intensity decreased with $t$.

If a release rate of the dye is limited by diffusion in the microparticle, the fluorescence intensity near the microparticle surface is smaller than that in the particle interior due to the concentration gradient in the microparticle. ${ }^{11}$ However, as shown in Fig. 1, the fluorescence intensity in the microparticle was almost constant at a $t$, indicating that the intraparticle diffusion rate is much greater than the release rate. These characteristic features were observed for the release of coumarin 102 from ODS-silica gel into water in the presence and absence of Triton X-100. In the present system, the intraparticle diffusion is not the rate-determining step of the dye release.

\section{Release rate of coumarin 102}

The total fluorescence intensity was calculated from the fluorescence depth profile. Figure 2 shows the time dependence of the total fluorescence intensity $\left(I_{\mathrm{t}}(t)\right)$ in the ODS-silica gel. The $I_{\mathrm{t}}(t)$ curve significantly depended on [Triton X-100] $]_{\mathrm{w}}$. In the perylene/ODS-silica gel system, perylene as a waterinsoluble dye was not released from ODS-silica gel in the absence of Triton X-100. ${ }^{17}$ However, coumarin 102 is slightly soluble in water so that the dye was slowly released from ODSsilica gel without Triton X-100. 


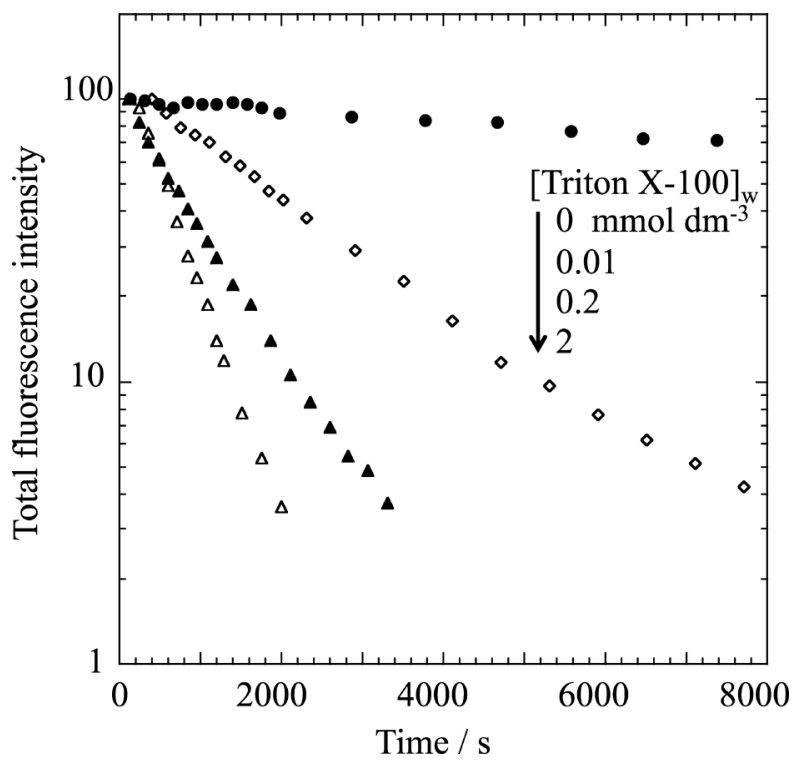

Fig. 2 Time dependence of total fluorescence intensity of coumarin 102 in single ODS-silica gel microparticles injected into aqueous Triton X-100 solutions of $0(d=40 \mu \mathrm{m}), 0.01(d=38 \mu \mathrm{m}), 0.2(d=$ $42 \mu \mathrm{m})$, and $2 \mathrm{mmol} \mathrm{dm}^{-3}(d=39 \mu \mathrm{m})$.

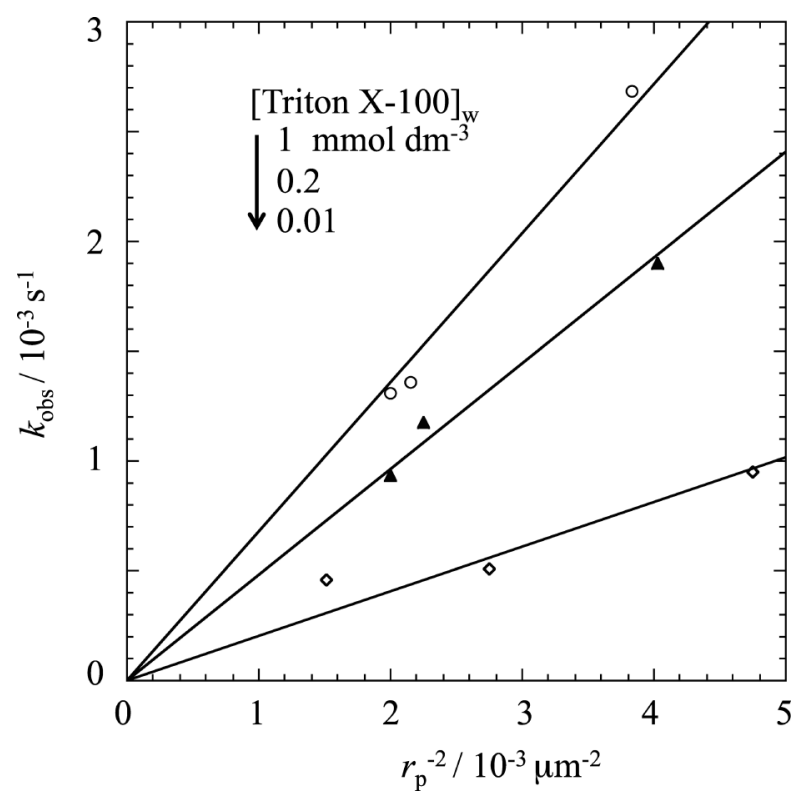

Fig. 3 Dependence of $k_{\mathrm{obs}}$ on $r_{\mathrm{p}}{ }^{-2}$ for single microparticles injected into aqueous Triton X-100 solutions of $0.01,0.2$, and $1 \mathrm{mmol} \mathrm{dm}^{-3}$.

Since logarithmic $I_{\mathrm{t}}(t)$ linearly decreased with $t$ (Fig. 2), $I_{\mathrm{t}}(t)$ was analyzed on the basis of the first-order reaction type equation: $I_{\mathrm{t}}(t)=I_{0} \exp \left(-k_{\mathrm{obs}} t\right)$, where $k_{\mathrm{obs}}$ and $I_{0}$ are the observed release rate constant and the total fluorescence intensity at $t=0$, respectively. Figure 3 shows the microparticle radius $\left(r_{\mathrm{p}}\right)$ dependence of $k_{\mathrm{obs}}$. When $k_{\mathrm{obs}}$ is directly proportional to $r_{\mathrm{p}}^{-1}$, the release rate is governed by a process at the spherical microparticle surface. ${ }^{24-26}$ In the perylene/ODS-silica gel system in the presence of Triton X-100, $k_{\mathrm{obs}}$ measured by absorption microspectroscopy of single microparticles was directly proportional to $r_{\mathrm{p}}^{-1}$, and the release of perylene from ODS-silica gel was observed at [Triton $\mathrm{X}-100]_{\mathrm{w}}$ greater than the critical micelle concentration $\left(\mathrm{cmc}, 0.2 \mathrm{mmol} \mathrm{dm}^{-3}\right){ }^{27,28}$ Therefore, the

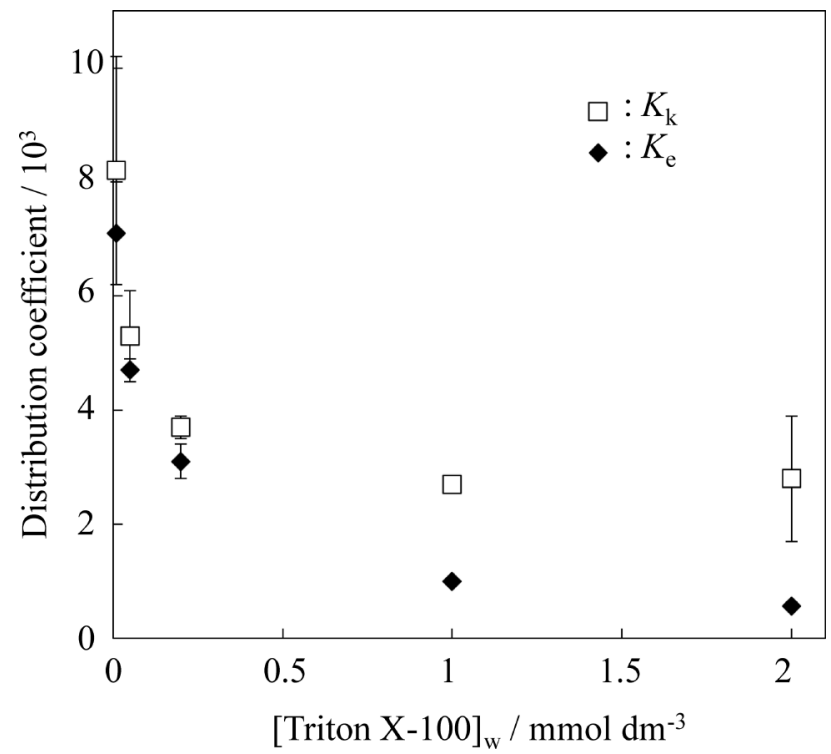

Fig. 4 Relationship between distribution coefficient $\left(K_{\mathrm{k}}\right.$ and $\left.K_{\mathrm{e}}\right)$ and [Triton X-100] .

process at the spherical surface was expected to be micellemediated solubilization of perylene by Triton X-100. ${ }^{17}$ In the coumarin 102/ODS-silica gel system, however, the release of the dye was observed at [Triton X-100] $]_{\mathrm{w}}$ smaller than $\mathrm{cmc}$, and $k_{\mathrm{obs}}$ was not directly proportional to $r_{\mathrm{p}}{ }^{-1}$. Therefore, the ratedetermining step of the release for coumarin 102 is not the process at the spherical microparticle surface. As shown in Fig. 3, $k_{\mathrm{obs}}$ is expected to be directly proportional to $r_{\mathrm{p}}{ }^{-2}$.

When a mass transfer rate constant between a spherical microparticle and the surrounding solution is directly proportional to $r_{\mathrm{p}}^{-2}$, the rate-determining step has been reported to be diffusion in the solution near the microparticle (external diffusion). According to the external diffusion-limited model, $k_{\mathrm{obs}}$ is given by the equation: $k_{\mathrm{obs}}=3 D_{\mathrm{w}} /\left(r_{\mathrm{p}}^{2} K_{\mathrm{k}}\right)$, where $D_{\mathrm{w}}$ is the diffusion coefficient of coumarin 102 in water, and $K_{\mathrm{k}}$ is the distribution coefficient of the dye between the ODS-silica gel and water. ${ }^{24,26}$ In this model, contribution of another phase such as a micelle phase is excluded. Using the known $D_{\mathrm{w}}$ value $\left(6 \times 10^{-6} \mathrm{~cm}^{2} \mathrm{~s}^{-1}\right){ }^{29} K_{\mathrm{k}}$ was calculated from the slope of the $k_{\mathrm{obs}}$ versus $r^{-2}$ plot (Fig. 3). As shown in Fig. 4, the calculated $K_{\mathrm{k}}$ decreased with the increasing [Triton $\mathrm{X}-100]_{\mathrm{w}}$ and remained constant at $\sim 3 \times 10^{3}$.

A distribution coefficient of coumarin 102 between the ODSsilica gel and the water phase $\left(K_{\mathrm{e}}\right)$ was experimentally determined from the dye concentrations in a single microparticle $\left(C_{\mathrm{p}}\right)$ and the water phase $\left(C_{\mathrm{w}}\right)$ at the distribution equilibrium (Fig. 4). In this case, $K_{\mathrm{e}}$ is defined as $K_{\mathrm{e}}=C_{\mathrm{p}} /\left(\varepsilon_{\mathrm{p}} C_{\mathrm{w}}\right)$, where $\varepsilon_{\mathrm{p}}$ is the porosity of the ODS-silica gel $(0.69) .{ }^{15}$ The $K_{\mathrm{e}}$ value agreed very well with $K_{\mathrm{k}}$ at [Triton X-100] $]_{\mathrm{w}}$ smaller than $\sim 0.2 \mathrm{mmol} \mathrm{dm}^{-3}$ within the experimental errors. However, $K_{\mathrm{e}}$ was much less than $K_{\mathrm{k}}$ at [Triton X-100] $\mathrm{w}=1$ and $2 \mathrm{mmol} \mathrm{dm}^{-3}$. At [Triton $\mathrm{X}-100]_{\mathrm{w}}$ greater than $\mathrm{cmc}\left(0.2 \mathrm{mmol} \mathrm{dm}^{-3}\right)$, the micelle exists in the water phase. At [Triton $X-100]_{\mathrm{w}}=1$ and $2 \mathrm{mmol} \mathrm{dm}^{-3}, C_{\mathrm{w}}$ was the sum of the amount of the dye molecules in both the water and the micelles, so the $K_{\mathrm{e}}$ value will be less than $K_{\mathrm{k}}$. Since the concentration of monomer Triton $\mathrm{X}-100$ in the water is $\sim 0.2 \mathrm{mmol} \mathrm{dm}^{-3}$ at [Triton $\left.\mathrm{X}-100\right]_{\mathrm{w}}>$ $\mathrm{cmc}$, the estimated $K_{\mathrm{k}}$ value will be almost constant at [Triton $\mathrm{X}-100]_{\mathrm{w}}>\sim 0.2 \mathrm{mmol} \mathrm{dm}^{-3}$. We consider that $k_{\mathrm{obs}}$ can be successfully analyzed by the external diffusion-limited model, 
and the rate-determining step of the coumarin 102 release from the ODS-silica gel is the external diffusion.

\section{External and intraparticle diffusion}

The external $\left(v_{\mathrm{w}}\right)$ or intraparticle diffusion rate $\left(v_{\mathrm{p}}\right)$ in the vicinity of the microparticle surface is given by $v_{\mathrm{w}}=D_{\mathrm{w}} C_{\mathrm{w}} / r_{\mathrm{p}}$ or $v_{\mathrm{p}}=D_{\mathrm{p}} C_{\mathrm{p}} / r_{\mathrm{p}}$, respectively. In the present system, $v_{\mathrm{p}} / v_{\mathrm{w}}=D_{\mathrm{p}} \varepsilon_{\mathrm{p}} K_{\mathrm{e}} /$ $D_{\mathrm{w}}$ will be much greater than unity, namely, $D_{\mathrm{p}} \gg D_{\mathrm{w}} /\left(\varepsilon_{\mathrm{p}} K_{\mathrm{e}}\right)$. As $K_{\mathrm{e}}=5 \times 10^{3}, D_{\mathrm{p}}$ is estimated to be much greater than $2 \times$ $10^{-9} \mathrm{~cm}^{2} \mathrm{~s}^{-1}$. According to the pore and surface diffusion model, on the other hand, $D_{\mathrm{p}}$ is given by the equation for $K_{\mathrm{e}} \gg>1: D_{\mathrm{p}}=$ $D_{\mathrm{w}} H /\left\{\tau_{\mathrm{p}}\left(1+K_{\mathrm{e}}\right)\right\}+D_{\mathrm{s}} / \tau_{\mathrm{s}}, 14,30$ where $H$ is the hindrance parameter and estimated to be 0.69 by the Renkin equation as the coumarin 102 diameter is $1 \mathrm{~nm} .{ }^{31} D_{\mathrm{s}}$ is the surface diffusion coefficient of coumarin 102 . The value of $\tau_{\mathrm{p}}$ or $\tau_{\mathrm{s}}$ is the tortuosity for the pore or surface diffusion in the ODS-silica gel, respectively, and has been reported to be $\sim 2 .^{32}$ The first and second terms of the equation for $D_{\mathrm{p}}$ correspond to the pore and surface diffusion, respectively. If contribution of the surface diffusion is negligibly small, $D_{\mathrm{p}}$ is calculated to be $\sim 4 \times 10^{-10} \mathrm{~cm}^{2} \mathrm{~s}^{-1}$ as $K_{\mathrm{e}}=5 \times 10^{3}$. The $D_{\mathrm{p}}$ value is much smaller than that estimated from $v_{\mathrm{p}} / v_{\mathrm{w}}$. Therefore, we consider that the $D_{\mathrm{s}}$ value is much greater than $2 \times 10^{-9} \mathrm{~cm}^{2} \mathrm{~s}^{-1}$.

\section{Conclusions}

Release of coumarin 102, as a poorly water-soluble organic compound, from the ODS-silica gel was kinetically analyzed using single microparticle injection and confocal fluorescence microspectroscopy. By the time dependence of the fluorescence depth profile in the single microparticle, we could directly demonstrate that the release rate is not governed by the intraparticle diffusion. The $r_{\mathrm{p}}$ dependence of $k_{\mathrm{obs}}$ could be analyzed on the basis of the diffusion-limited model between the microparticle surface and the surrounding solution phase. We concluded that the rate-determining step is the external diffusion and the rate of the surface diffusion in the microparticle is much faster than that of the external diffusion. The confocal fluorescence microspectroscopy combined with the single microparticle injection technique will be indispensable for the kinetic analysis of mass transfer in the porous microparticle system.

\section{Acknowledgements}

This work was supported by JSPS KAKENHI Grant No. 22550069. The authors would like to thank the Chemical Analysis Division and Open Facility, Research Facility Center for Science and Technology, University of Tsukuba, for allowing us to use the confocal fluorescence microscope (Olympus, FV 1000-D).

\section{References}

1. D. M. Ruthven, "Principles of Adsorption and Adsorption
Processes", 1984, Wiley, New York.

2. N. Wu, M. A. Hubbe, O. J. Rojas, and S. Park, BioResources, 2009, 4, 1222.

3. H. Yoshida, M. Yoshikawa, and T. Kataoka, AIChE J., 1994, 40, 2034.

4. D. M. Ruthven, Chem. Eng. Sci., 2004, 59, 4531.

5. K. Miyabe and M. Suzuki, AIChE J., 1995, 41, 548.

6. K. Nakatani and T. Sekine, Langmuir, 2000, 16, 9256.

7. R. Bujalski and F. F. Cantwell, J. Chromatogr. A, 2004, 1048, 173.

8. A. Yamaguchi, M. M. Mekawy, Y. Chen, S. Suzuki, K. Morita, and N. Teramae, J. Phys. Chem. B, 2008, 112, 2024.

9. N. Kameta, H. Minamikawa, Y. Someya, H. Yui, M. Masuda, and T. Shimizu, Chem. Eur. J., 2010, 16, 4217.

10. F. Roncaroli and M. A. Blesa, J. Colloid Interface Sci., 2011, 356, 227.

11. H.-B. Kim, M. Hayashi, K. Nakatani, N. Kitamura, K. Sasaki, J. Hotta, and H. Masuhara, Anal. Chem., 1996, 68, 409.

12. M. D. Ludes, S. R. Anthony, and M. J. Wirth, Anal. Chem., 2003, 75, 3073.

13. T. Sekine and K. Nakatani, Langmuir, 2002, 18, 694.

14. T. Sekine and K. Nakatani, Chem. Lett., 2004, 33, 600.

15. H. Kakizaki, C. Aonuma, and K. Nakatani, J. Colloid Interface Sci., 2007, 307, 166.

16. K. Nakatani, K. Kobayashi, and A. Haga, Anal. Sci., 2008, 24, 377.

17. K. Nakatani, M. Miyanaga, and Y. Kawasaki, Anal. Sci., 2011, 27, 1253.

18. M. Koshioka, H. Misawa, K. Sasaki, N. Kitamura, and H. Masuhara, J. Phys. Chem., 1992, 96, 2909.

19. K. Nakatani, H. Misawa, K. Sasaki, N. Kitamura, and H. Masuhara, J. Phys. Chem., 1993, 97, 1701.

20. D. Grasso, K. Subramaniam, J. J. Pignatello, Y. Yang, and D. Ratte, Colloids Surf. A, 2001, 194, 65.

21. R. Sharmin, M. A. Ioannidis, and R. L. Legge, J. Contam. Hydrol., 2006, 82, 145.

22. J. K. Strasters, E. D. Breyer, A. H. Rodgers, and M. G. Khaledi, J. Chromatogr., 1990, 511, 17.

23. T. Okada, Bunseki Kagaku, 1998, 47, 1069.

24. K. Nakatani, T. Uchida, N. Kitamura, and H. Masuhara, J. Electroanal. Chem., 1994, 375, 383.

25. K. Nakatani, K. Chikama, H.-B. Kim, and N. Kitamura, Chem. Phys. Lett., 1995, 237, 133.

26. K. Nakatani, M. Wakabayashi, K. Chikama, and N. Kitamura, J. Phys. Chem., 1996, 100, 6749.

27. R. J. Robson and E. A. Dennis, J. Phys. Chem., 1977, 81, 1075 .

28. A. Patist, J. R. Kanicky, P. K. Shukla, and D. O. Shah, J. Colloid Interface Sci., 2002, 245, 1.

29. A. K. Mandal, D. K. Das, A. K. Das, S. S. Monjumdar, and K. Bhattschryya, J. Phys. Chem. B, 2011, 115, 10456.

30. R. Bujalski and F. F. Cantwell, Anal. Chem., 2006, 78, 1593.

31. W. M. Deen, AIChE J., 1987, 33, 1409.

32. R. Bujalski and F. F. Cantwell, Langmuir, 2001, 17, 7710. 